American Journal of Applied Sciences 9 (4): 553-556, 2012

ISSN 1546-9239

(C) 2012 Science Publications

\title{
Role of Endometrial Thickness on Intracytoplasmic Sperm Injection
}

\author{
Mohammed Al-Sunaidi \\ Department of Obstetrics and Gynecology, \\ College of Medicine, King Khalid University, ABHA, P.O. Box 641, Saudi Arabia
}

\begin{abstract}
Problem statement: Endometrial receptivity is a key factor in successful outcome of in vitro fertilization. The endometrial receptivity is determined clinically by estimation of endometrial thickness and pattern by ultrasonography. This study was performed to evaluate the overall effect of endometrial thickness on pregnancy rate, multiple pregnancy rate and miscarriage rate in infertile women undergoing Intracytoplasmic Sperm Injection (ICSI) cycles at Mustasharak medical center, ABHA, Saudi Arabia. Approach: A prospective observational study at Mustasharak assisted reproduction center, ABHA, Saudi Arabia, was performed for women who underwent ICSI between Jan and Dec 2010. On the day of oocytes retrieval; after a true ultrasound longitudinal view of the uterus had been obtained, the endometrial thickness was measured as the maximum thickness between the highly reflective interfaces of the endometrial-myometrial junction. Results: 409 ICSI cycles were included in the study. The highest pregnancy rate was seen in patients who had an endometrial thickness of more than $12 \mathrm{~mm}$, while those who had an endometrial thickness of less than $8 \mathrm{~mm}$ had the worst pregnancy rate. Type B endometrial morphology (trilaminar) had a significant higher pregnancy rate than type A endometrial morphology (non trilaminar). Conclusion: This study demonstrated a clear relationship between endometrial thickness on the day of oocytes collection and pregnancy rates following ICSI.
\end{abstract}

Key words: Intracytoplasmic Sperm Injection (ICSI), endometrial thickness, infertility, assisted reproduction, Assisted Reproduction Technology (ART)

\section{INTRODUCTION}

The overall success in ICSI cycles depends on female partner age, embryos quality and endometrial receptivity which lead to successful implantation and outcome. Ultrasonographic examination has been routinely performed in Assisted Reproduction Technology (ART) treatments because of the accurate evaluation and noninvasive detection.

Successful implantation depends on a close dialog between the blastocyst and the receptive endometrium. Different strategies have been developed to evaluate endometrial receptivity, such as the histologic dating of an endometrial biopsy (Sterzik et al., 2000), endometrial cytokines in uterine flushing (LedeeBataille et al., 2002), the genomic study of a timed endometrial biopsy (Horcajadas et al., 2007) or more commonly ultrasound examination of the endometrium. Following stimulation of ovarian hormones, the changes in endometrial structure during the menstrual cycle can be identified easily by ultrasound examination (Killick, 2007). In an IVF/ICSI procedure, hCG is used as a substitute for the natural Luteinizing Hormone ( $\mathrm{LH}$ )-surge to trigger the final maturity of the oocyte. Evaluation of endometrium on the day of $\mathrm{hCG}$ administration is of great clinical importance (Friedler et al., 1996; Oliveira et al., 1997). Several studies have demonstrated the existence of a correlation between endometrial characteristics and pregnancy rate in IVF/ICSI patients (Noyes et al., 1995; Richter et al., 2007; Al-Ghamdi et al., 2008). However, the correlation proposed in these studies has not been universally accepted (Yuval et al., 1999; Rashidi et al., 2004). There is also no consensus on whether the endometrial ultrasound characteristics can predict the pregnancy outcome in IVF/ICSI treatment.

A no triple-line endometrial pattern seems to be a prognostic sign of a less favorable outcome, while a triple-line pattern appear to be associated with conception (Gonen et al., 1991; Check et al., 1993; Jarvela et al., 2005).

In this study, we examined the correlation between endometrial thickness and pattern and ICSI outcome. Our objective is to investigate whether analysis of endometrial thickness and pattern would improve the prediction of clinical outcome compared to the analysis of thickness or pattern separately. 
Am. J. Applied Sci., 9 (4): 553-556, 2012

\section{MATERIALS AND METHODS}

A prospective observational study at Mustasharak assisted reproduction center, ABHA, Saudi Arabia, was performed for women who underwent ICSI between Jan and Dec 2010. The study protocol was approved by the Institutional Review Board before beginning of the study and it was performed according to principles of Helsinki declaration.

Exclusion criteria for the study included failure of all eggs to be fertilized, poor quality embryos at the day of the transfer, or elective cryopreservation of all embryos. All study subjects underwent controlled ovarian hyperstimulation (with a long protocol regimen in all cases, including down-regulation with GnRH analogue and ovarian stimulation with recombinant FSH and transvaginal oocyte retrieval after the injection of 10,000 IU of HCG. ICSI was performed with the husband's spermatozoa according to the routine protocols at our center and sequential culture media from Vitrolife (IVF-20, G1.2 and G2.2, Scandinavian IVF Science AB, Göteborg, Sweden) were used in all cases.

On the day of oocytes retrieval; after a true ultrasound longitudinal view of the uterus had been obtained, the endometrial thickness was measured as the maximum thickness between the highly reflective interfaces of the endometrial-myometrial junction. The measurement included both layers of the endometrium. The surrounding low-amplitude echo layer was excluded, because it represents the inner layers of the myometrium. Endometrial morphology was classified as types A (non triple line), B (triple-line).

Embryologists in the IVF lab evaluated the patients' embryos on day 3 after retrieval and the grading of each embryo was documented.

The primary outcome was clinical pregnancy, defined as the presence of fetal heart activity by ultrasound at 6-7 weeks' gestation.

Secondary outcome included miscarriage rate, multiple pregnancy rate and ectopic pregnancy rate.

Statistical analysis used Student's $t$ test and chi-squared test as applicable. Analysis of the data was performed using IBM® SPSS $®$ Statistics 16 software.

\section{RESULTS}

A total of 409 ICSI cycles were included in the study with an overall pregnancy rate of $42.8 \%$.

Our patients had the following primary etiologies for their infertility: male factor $(49.8 \%)$, anovulation (26.9\%), tubal factor $(8.7 \%)$, unexplained $(10.7 \%)$, endometriosis $(1 \%)$ and combined factors $(2.9 \%)$.
Table 1: Patient and cycle characteristics for pregnant and non pregnant women

\begin{tabular}{lcr}
\hline Characteristic & Pregnant & Non pregnant \\
\hline Number of cycles & 175 & 234.0 \\
Age (20-25 years) & 22 & 29.0 \\
(25-30 years) & 84 & 88.0 \\
(30-35 years) & 43 & 68.0 \\
(35-37 years) & 11 & 30.0 \\
(37-40 years) & 15 & 19.0 \\
Type of infertility & & \\
Tubal disease & 15 & 20.0 \\
Male factor & 80 & 123.0 \\
Anovulation & 52 & 58.0 \\
Endometriosis & 1 & 3.0 \\
Unexplained & 22 & 20.0 \\
combined & 3 & 9.0 \\
Baseline FSH & 5.7 & 5.2 \\
\hline
\end{tabular}

Table 2: Rates of clinical pregnancy, spontaneous abortion, ectopic, multiple and biochemical pregnancies

\begin{tabular}{llcccr}
\hline $\begin{array}{l}\text { Endometrial } \\
\text { Thickness }\end{array}$ & $\begin{array}{l}\text { clinical } \\
\text { pregnancy }(\%)\end{array}$ & $\begin{array}{c}\text { Multiple } \\
\text { pregnancy }\end{array}$ & $\begin{array}{c}\text { Spontaneous } \\
\text { abortion }\end{array}$ & \multicolumn{2}{c}{$\begin{array}{c}\text { Biochemical Ectopic } \\
\text { pregnancy }\end{array}$} \\
\hline$<6$ pregnancy \\
\hline$<6 \mathrm{~mm}$ & 0 & 0 & 0 & 0 & 0 \\
$6-8 \mathrm{~mm}$ & 0 & 0 & 0 & 3 & 0 \\
$8-10 \mathrm{~mm}$ & $22(31 \%)$ & 10 & 0 & 9 & 0 \\
$10-12 \mathrm{~mm}$ & $38(41 \%)$ & 14 & 5 & 13 & 1 \\
$12-14 \mathrm{~mm}$ & $67(53 \%)$ & 24 & 24 & 8 & 0 \\
$>14 \mathrm{~mm}$ & $15(53 \%)$ & 8 & 2 & 0 & 1 \\
Total & 142 & 56 & 31 & 33 & 2 \\
\hline
\end{tabular}

There were no significant difference between the non pregnant and pregnant group of patients in term of cause of infertility and the base line FSH level. Characteristics of both groups are shown in Table 1.

The highest pregnancy rate was seen in patients who had an endometrial thickness of more than $12 \mathrm{~mm}$ $(53 \%)$ in different age groups while those who had an endometrial thickness of less than $8 \mathrm{~mm}$ had the worst pregnancy rate; these findings are illustrated in Table 2.

It was found that type B endometrial morphology had a significant higher pregnancy rate than type A endometrial morphology with a $\mathrm{P}$ value equal to 0.00001 and $95 \%$ CI ( -0.57 to -0.19$)$.

Multiple pregnancy rate was highest in those patients who had an endometrial thickness of more than $14 \mathrm{~mm}$, while the highest rate of spontaneous miscarriage was seen in patients who had an endometrial thickness between 12 and $14 \mathrm{~mm}$. Rates of spontaneous abortion, biochemical, ectopic, clinical and multiple pregnancies are shown in Table 2.

\section{DISSCUSION}

Our results agree with other studies that report an improved pregnancy rate with increased endometrial thickness (Glissant et al., 1985; Gonen et al., 1989; Kovacs et al., 2003; Abdalla et al., 1994; Zenke and Chetkowski, 2004). 
There is a steady and gradual increase in pregnancy rates as endometrial thickness increases, throughout the range of endometrial thicknesses observed in the study population. This clear relationship between endometrial thickness and pregnancy provides additional evidence to suggest that endometrial thickness is a useful indicator of endometrial receptivity.

There is a lack of agreement with regard to the minimum endometrial thickness required for successful pregnancy. In one study, no pregnancies occurred when the endometrial thickness was less than $7 \mathrm{~mm}$ (Oliveira et al., 1997), while in our study there were no pregnancies in patients who had an endometrial thickness of less than $6 \mathrm{~mm}$ on the day of oocytes collection which is consistent with other study that have demonstrated that a minimum thickness of $6 \mathrm{~mm}$ is acceptable as a prerequisite for implantation (Gonen $e t$ al., 1989; Shapiro et al., 1993; Coulam et al., 1994).

Another important finding observed in this study is the significantly higher pregnancy rate in triple-line endometrium compared to non-triple-line endometrium and this finding was also observed in another study (Chen et al., 2010).

We found no indication of reduced pregnancy rates or increased spontaneous abortion rates with a very thick endometrial lining $(>14 \mathrm{~mm})$, as reported by others (Weissman et al., 1999). They speculated that, if their conclusions were validated with a larger database, elective embryo cryopreservation for transfer in a later cycle might be advisable for patients with unusually thick linings. Our results instead suggest that clinical pregnancy are highest for patients with the thickest linings and are more consistent with results of other studies finding no reduction in pregnancy rates with very thick linings (Zhang et al., 2005; Yakin et al., 2000; Dietterich et al., 2002; Yoeli et al., 2004).

Continued use of transvaginal ultrasound to evaluate endometrial thickness and the change occurring during ovarian stimulation can aid providers in counseling patients and predicting ICSI success. An increase in endometrial response seems to prognosticate better ICSI success. Endometrial receptivity is still difficult to prognosticate. It is unclear if the improved ICSI success is because of a more responsive or sensitive endometrial lining or if the responsiveness of the endometrial lining is only a marker of better gonadotropin stimulation of the ovary with downstream effects on the endometrium.

\section{CONCLUSION}

In conclusion, this study demonstrated a clear relationship between endometrial thickness on the day of oocytes collection and pregnancy rates following ICSI.

When a thinner endometrium $(<6 \mathrm{~mm})$ and no triple-line endometrial pattern coexist in an IVF/ICSI candidate, cryopreservation might be a better option to be recommended. Because endometrial thickness $<6$ $\mathrm{mm}$ with no-triple line pattern was seen in only $0.005 \%$ of cycles in our study, further study is needed to make a definitive conclusion. If a thinner endometrium with a good texture (triple-line) is present, other prognostic factors, such as embryo quality, should be taken into consideration. Regardless of the endometrial pattern, a thicker endometrium $(>14 \mathrm{~mm})$ did not have an adverse effect on the clinical outcome. Combined analysis of endometrial thickness and pattern on the day of oocytes collection could be more valuable than the separate analyses.

\section{REFERENCES}

Abdalla, H.I., A.A. Brooks, M.R. Johnson, A. Kirkland and A. Thomas et al., 1994. Endometrial thickness: A predictor of implantation in ovum recipients? Hum. Reprod., 9: 363-365.

Al-Ghamdi, A., S. Coskun, S. Al-Hassan, R. AL-Rejjal and K. Awartani, 2008. The correlation between endometrial thickness and outcome of in vitro fertilization and embryo transfer (IVF-ET) outcome. Reprod. Biol. Endocrinol., 6: 37-37. DOI:10.1186/1477-7827-6-37

Check, J.H., D. Lurie, C. Dietterich, C. Callan and A. Baker, 1993. Adverse effect of a homogeneous hyperechogenic endometrial sonographic pattern, despite adequate endometrial thickness on pregnancy rates following in-vitro fertilization. Hum. Reprod., 8: 1293-1296.

Chen, S.L. et al., 2010. Combined analysis of endometrial thickness and pattern in predicting outcome of in vitro fertilization and embryo transfer: a retrospective cohort study. Reproductive Biology Endocrinology., 8: 30-30. DOI: 10.1186/1477-7827-8-30

Coulam, C.B., M. Bustillo, D.M. Soenksen and S. Britten, 1994. Ultrasonographic predictors of implantation after assisted reproduction. Fertil. Steril., 62: 1004-1010. PMID: 7926110

Dietterich, C., J.H. Check, J.K. Choe, A. Nazari and D. Lurie, 2002. Increased endometrial thickness on the day of human chorionic gonadotropin injection does not adversely affect pregnancy or implantation rates following in vitro fertilizationembryo transfer. Fertil. Steril., 77: 781-786.

Friedler, S., J.G. Schenker, A. Herman and A. Lewin, 1996. The role of ultrasonography in the evaluation of endometrial receptivity following assisted reproductive treatments: A critical review. Hum Reprod. Update., 2: 323-334. DOI: 10.1093/humupd/2.4.323 
Glissant, A., J. De Mouzon and R. Frydman, 1985. Ultrasound study of the endometrium during in vitro fertilization cycles. Fertil. Steril., 44: 786-90. PMID: 4076435

Gonen, Y., I. Calderon, M. Dirnfeld and H. Abramovici, 1991. The impact of sonographic assessment of the endometrium and meticulous hormonal monitoring during natural cycles in patients with failed donor artificial insemination. Ultrasound Obstet. Gynecol., 1: 122-126. DOI: 10.1046/j.1469-0705.1991.01020122.x

Gonen, Y., R.F. Casper, W. Jacobson and J. Blankier, 1989. Endometrial thickness and growth during ovarian stimulation: A possible predictor of implantation in in vitro fertilization. Fertil. Steril., 52: 446-450. PMID: 2673844

Horcajadas, J.A., A. Pellicer and C. Simón, 2007. Wide genomic analysis of human endometrial receptivity: New times, new opportunities. Hum Reprod Update, 13: 77-86. DOI: 10.1093/humupd/dml046

Jarvela, I.Y., P. Sladkevicius, S. Kelly, K. Ojha and S. Campbell et al., 2005. Evaluation of endometrial receptivity during in-vitro fertilization using threedimensional power Doppler ultrasound. Ultrasound Obstet. Gynecol., 26: 765-769. DOI: 10.1002/uog.2628

Killick, S.R., 2007. Ultrasound and the receptivity of the endometrium. Reprod Biomed. Online, 15: 6367. PMID: 17623538

Kovacs, P., S. Matyas, K. Boda and S.G. Kaali, 2003. The effect of endometrial thickness on IVF/ICSI outcome. Hum. Reprod., 18: 2337-2341. DOI: 10.1093/humrep/deg461

Ledee-Bataille, N., G. Laprée-Delage, J.L. Taupin, S. Dubanchet and R. Frydman et al., 2002. Concentration of Leukaemia Inhibitory Factor (LIF) in uterine flushing fluid is highly predictive of embryo implantation. Hum Reprod, 17: 213218. DOI: $10.1093 /$ humrep/17.1.213

Noyes, N., H.C. Liu, K. Sultan, G. Schattman and Z. Rosenwaks, 1995. Endometrial thickness appears to be a significant factor in embryo implantation in in-vitro fertilization. Hum. Reprod., 10: 919-922. PMID: 7650143

Oliveira, J.B.A., R.L.R. Baruffi, A.L. Mauri, C.G. Petersen and M.C. Borges et al., 1997. Endometrial ultrasonography as a predictor of pregnancy in an in-vitro fertilization programme after ovarian stimulation and gonadotrophin releasing hormone and gonadotrophins. Hum Reprod., 12: 2515-2518. DOI: $10.1093 /$ humrep/12.11.2515
Rashidi, B.H., M. Sadeghi, M. Jafarabadi and E.S.T. Nejad, 2004. Relationships between pregnancy rates following in vitro fertilization or intracytoplasmic sperm injection and endometrial thickness and pattern. Eur. J. Obstet Gynecol. Reprod. Biol., 120: 179-184. DOI: 10.1016/j.ejogrb.2004.08.016

Richter, K.S., K.R. Bugge, J.G. Bromer and M.J. Levy, 2007. Relationship between endometrial thickness and embryo implantation, based on 1,294 cycles of in vitro fertilization with transfer of two blastocyststage embryos. Fertil Steril, 87: 53-59.

Shapiro, H., C. Cowell and R.F. Casper, 1993. The use of vaginal ultrasound for monitoring endometrial preparation in a donor oocyte program. Fertil. Steril., 59: 1055-1058. PMID: 8486173

Sterzik, K, Abt M, Grab D, Schneider V, Strehler E. Predicting the histologic dating of an endometrial biopsy specimen with the use of Doppler ultrasonography and hormone measurements in patients undergoing spontaneous ovulatory cycles. Fertil Steril., 73:94-98. PMID: 10632420

Weissman, A., L. Gotlieb and R.F. Casper, 1999. The detrimental effect of increased endometrial thickness on implantation and pregnancy rates and outcome in an in vitro fertilization program. Fertil. Steril., 71: 147-149. PMID: 9935132

Yakin, K., C. Akarsu and S. Kahraman, 2000. Cycle lumping or--sampling a witches' brew? Fertil. Steril., 73: 175-175. PMID: 10632437

Yoeli, R., J. Ashkenazi, R. Orvieto, M. Shelef and B. Kaplan et al., 2004. Significance of increased endometrial thickness in assisted reproduction technology treatments. J. Assist. Reprod. Genet., 21: 285-289. PMID: 15568328

Yuval, Y., S. Lipitz, J. Dor and R. Achiron, 1999. The relationships between endometrial thickness and blood flow and pregnancy rates in in-vitro fertilization. Hum Reprod., 14: 1067-1071. DOI: 10.1093/humrep/14.4.1067

Zenke, U. and R.J. Chetkowski, 2004. Transfer and uterine factors are the major recipient-related determinants of success with donor eggs. Fertil. Steril., 82: 850-856. DOI: 10.1016/j.fertnstert.2004.03.057

Zhang, X., C.H. Chen, E. Confino, R. Barnes and M. Milad et al., 2005. Increased endometrial thickness is associated with improved treatment outcome for selected patients undergoing in vitro fertilizationembryo transfer. Fertil. Steril., 83: 336-340. DOI: 10.1016/j.fertnstert.2004.09.020 